\title{
Channel Properties of indoor part for high-speed train based on wideband channel measurement
}

\author{
Weihui Dong ${ }^{\dagger}$, Guangyi Liu ${ }^{\dagger}$, Li Yu ${ }^{\dagger}$, Haiyu Ding ${ }^{\dagger}$, and Jianhua Zhang ${ }^{\ddagger}$ \\ ${ }^{\dagger}$ China Mobile Research Institute \\ ${ }^{\ddagger}$ Key Laboratory of Universal Wireless Communication (Beijing University of Posts and \\ Telecommunications), Ministry of Education
}

\begin{abstract}
In this paper, the statistical channel properties and channel modeling of indoor part for high-speed train communication sysem is presented based on wideband channel measurement at $2.35 \mathrm{GHz}$. Two configurations of base station (BS) antennas, the omni-directional ceiling antenna and the planar antenna on the wall, are used in the measurement, in order to compare different channel characteristics and facilitate the future wideband system deployment. Channel properties, such as Path Loss (PL), Delay Spread (DS) and Ricean K-factor, are analyzed and modeled. The empirical log-distance PL models are derived. It is found that PL with planar antenna at $\mathrm{BS}$ is $10 \mathrm{~dB}$ bigger than that with omni-directional antenna. The latter is even smaller than the PL of the free space. The distributions of DS under these two configurations are both well fitted with the lognormal distribution, and the mean values of them are similar. However, K-factors in decibel are quite different, although both follow well with the normal distribution. The mean values of $\mathrm{K}$-factor with the omni-directional antenna and the planar antenna at $\mathrm{BS}$ are $\mathbf{1 0 . 4 1}$ $\mathrm{dB}$ and $4.09 \mathrm{~dB}$, respectively.
\end{abstract}

Keywords-high-speed train; Path Loss (PL); Delay Spread (DS); Ricean K-factor

\section{INTRODUCTION}

In railway communication system, GSM-R system [1-2] are commonly used to provide narrow band service, such as low rate data service and voice service, based on the track-side base stations. With growing demand for fast and safe transportation, high-speed trains are developing rapidly in China, with the speed of up to $350 \mathrm{~km} / \mathrm{h}$, or even up to $500 \mathrm{~km} / \mathrm{h}$. More and more people choose to travel by train. What they need in the train are varieties of services to pass the time, such as video service or games on the internet. Therefore, low rate service cannot meet the increasing demand and wideband data services are in great requirement.

Furthermore, wireless channel characteristics have already changed because of the high speed in motion, which means the performance of system simulation and product test based on the previous channel model will have a big gap with the practical result. In addition, in the traditional GSM-R system, terminals in the train receive signals directly from the track-side base stations. However, in the new high-speed train, this way means large penetration loss because of the much thicker train wall. Hence, in the new communication system, some additional node should be introduced, such as relay, as shown in Fig. 1. In this case, channel models for both base station to relay (outdoor part) and relay to terminal (indoor part) are needed.

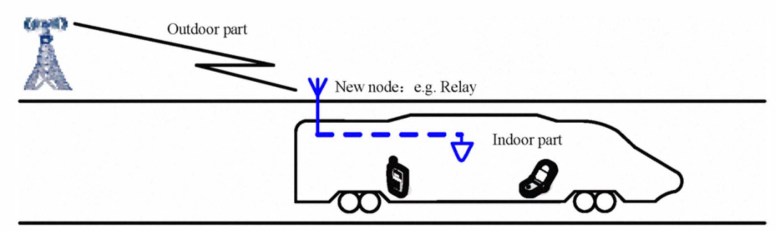

Fig. 1 New high-speed train communication system: consists of outdoor part and indoor part

A lot of research and product developing source have been attracted in the wideband high-speed train communication system recently. However, until now, there is seldom result on the wideband channel model for high speed train, which provides fundamental support for industry development. 3GPP defines a channel model for high speed train test [3-4]. This model is non-fading propagation channel model and consists of only one ray. It mainly focuses on Doppler effect on system performance and other channel characteristics are not considered.

For the first step, as described in this paper, we carried out channel measurement in the indoor part of high-speed train communication and channel properties are analyzed and modeled.

\section{MEASUREMENT SETUP AND ENVIRONMENT}

\section{A. Measurement Setup}

The wideband measurements were performed with $100 \mathrm{MHz}$

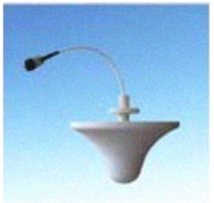

(a)

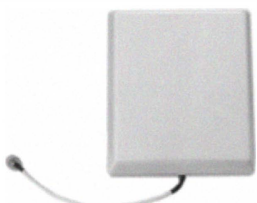

(b) (c)

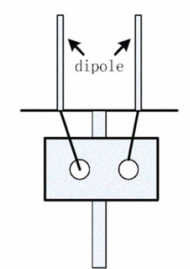

Fig. 2. Antennas at $2.35 \mathrm{GHz}$ : (a) omni-directional ceiling antenna. (b) Planar antenna. (C) Antennas at Rx.

bandwidth at $2.35 \mathrm{GHz}$ frequency using the Elektrobit Propsound [5] in the interior of high-speed train, which is made in Tangshan, China. Propsound is based on the principle of time 


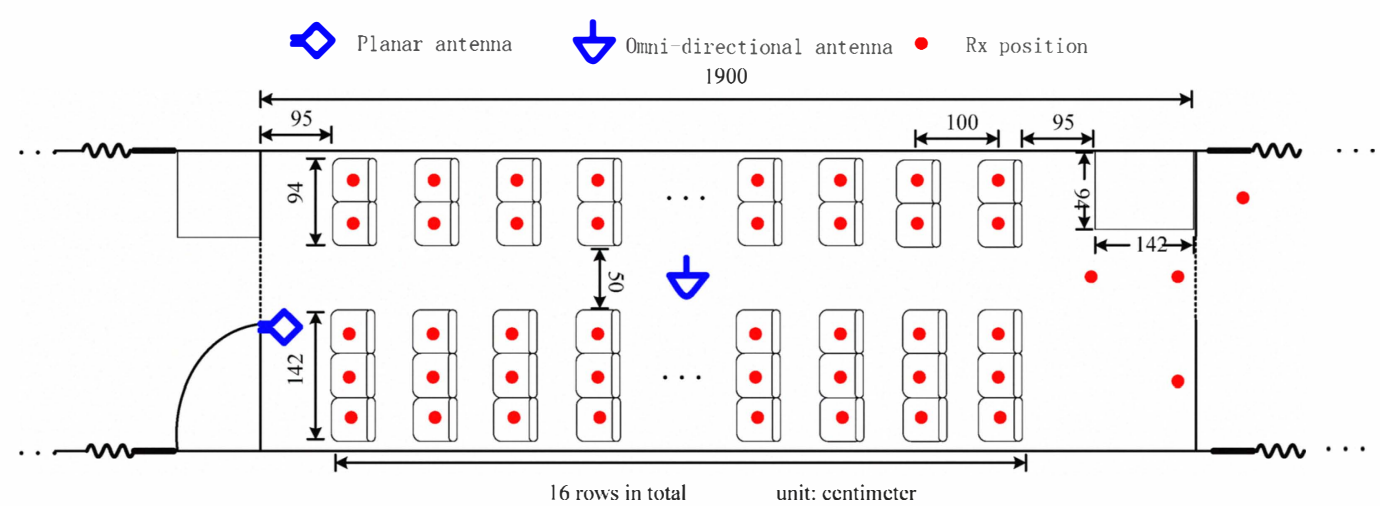

Fig. 3. Top view of high-speed train indoor geometry.

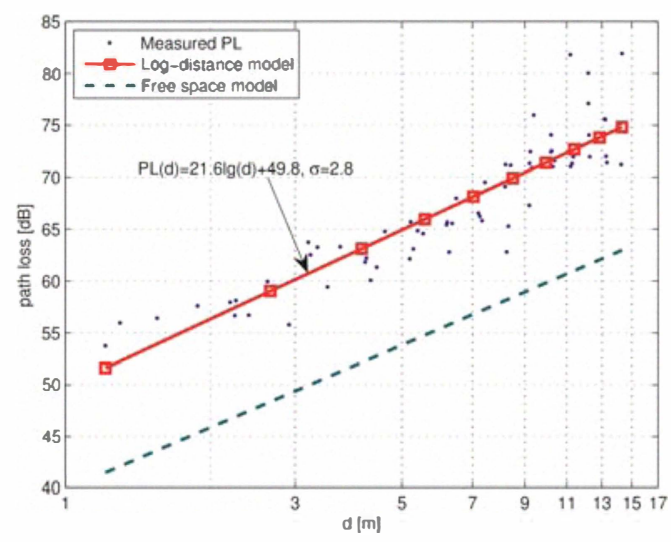

Fig. 4. Path Loss measurements and model when using planar antenna at Tx.

division multiplexing. A measurement snapshot refers to the time interval that all subchannels between transmitter (Tx) and receiver (Rx) antennas are sounded once. A snapshot is guaranteed within the coherence time of the channel to allow the channel parameter estimator to extract the channel parameters coherently.

There are two configurations of base station (BS) antennas in the measurement. One is omni-directional ceiling antenna and the other is planar antenna which is fixed on the wall, as shown in the Fig. 2(a) and Fig. 2(b), respectively. Note that these two antennas are already commonly applied in the indoor networks. Fig. 2(c) shows antenna array at $\mathrm{Rx}$, which consists of two dipole antennas with half lambda spacing. Since the result is targeted to facilitate future wideband system deployment in high-speed train, we design this antenna array at $\mathrm{Rx}$ with two dipole omni-directional elements in the measurement.

\section{B. Measurement Environment}

The measurements were carried out in the interior of high-speed train, whose geometry is shown in Fig. 3. The interior was enclosed by thick steel and several windows, which will cause lots of diffraction and reflection. During the measurements, omni-directional antenna at $\mathrm{Tx}$ was mounted on the ceiling with the height of about $2.3 \mathrm{~m}$, while planar antenna was fixed on the wall at the height of about $1.5 \mathrm{~m}$. The $\mathrm{Rx}$

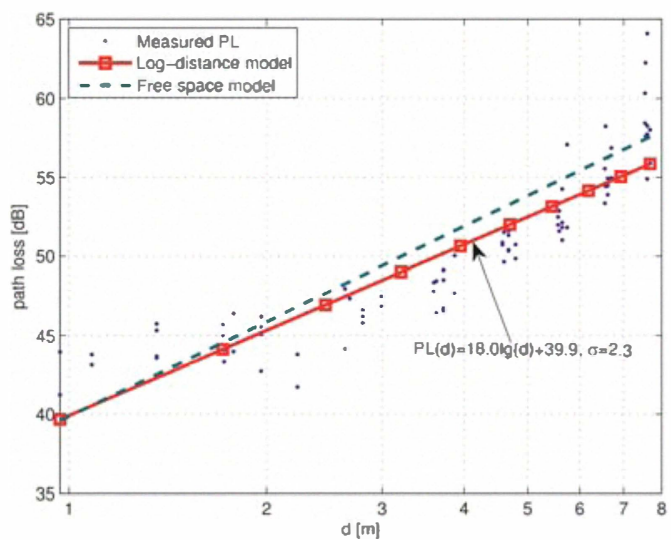

Fig. 5. Path Loss measurements and model when using omni-directional antenna at $\mathrm{Tx}$

antenna was set to $0.8 \mathrm{~m}$ at each measurement spot, which is noted as red spot in Fig. 3 . The $0.8 \mathrm{~m}$ height was designed to that when people played with cell phones sitting on the chair. At each measurement spot, 200 snapshots are measured and stored for post data processing.

\section{ANALYSIS RESULTS}

In this section, Path Loss, Delay Spread and Ricean K-factor are analyzed and modeled under two BS antenna configurations.

\section{A. Path Loss}

Path loss is defined as the ratio of the effective transmitted power to the received power, calibrating out cable losses, amplifier gains, and antenna gains. The measured PL values are calculated in decibels as

$$
\mathrm{PL}=-10\left(\sum_{\tau}\left|h_{a v}(\tau)\right|^{2}\right)+G_{T x}+G_{R x},
$$

Where

$$
h_{a v}(\tau)=E_{t}\{h(t, \tau)\}
$$

$G_{T x}$ and $G_{R x}$ are antenna gains at $\mathrm{Tx}$ and $\mathrm{Rx}$, respectively. $E_{t}\{\bullet\}$ means the average in time. Here, we average $h(t, \tau)$ in each measurement position. 


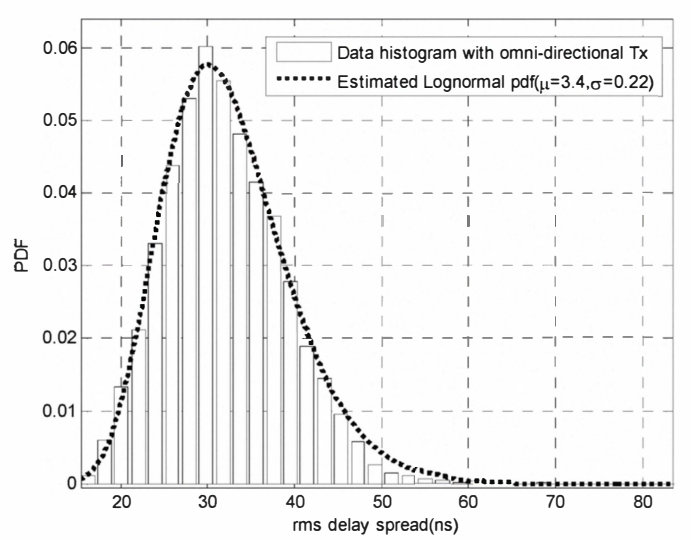

Fig. 6. Distribution of rms delay spread with omni-directional antenna at $\mathrm{Tx}$

The modeling method used for Path Loss is a linear curve fitting the decibel path loss to the decibel distance with a random variation. The empirical model of log-distance PL [6-7] is expressed as

$$
P L(d)=P L_{0}+10 n \log _{10}(d)+X_{\sigma}
$$

where $P L_{0}$ is the intercept, $d$ is the Tx-Rx separation distance in meters, and $n$ is the PL exponent dependent on the specific propagation environment indicating the rate at which PL increases with distance. In free space propagation, $\mathrm{n}$ equals 2 . $X_{\sigma}$ denotes shadow fading with standard deviation $\sigma$.

The values of $P L_{0}$ and $n$ are extracted by using the least square (LS) method. The modeling results are shown in Fig. 4 with planar antenna at $\mathrm{Tx}$, which is expressed as

$$
P L(d)=49.8+21.6 \log _{10}(d), \sigma=2.8 .
$$

And Fig. 5 shows the results with omni-directional antenna at Tx as

$$
P L(d)=39.9+18.0 \log _{10}(d), \sigma=2.3
$$

It can be seen that PL with planar antenna is $10 \mathrm{~dB}$ bigger than that with omni-directional antenna in average, which means in order to get the same receive power, the transmitting power with planar antenna is 10 times of that with omni-directional antenna at $\mathrm{Tx}$. One reason is that the line-of-sight (LOS) route between $\mathrm{Tx}$ and $\mathrm{Rx}$ is more easily to be blocked by the chair and other scatters in the case of planar antenna.

Another interesting phenomena is that PL with omni-directional antenna at $\mathrm{Tx}$ is even less than that of free space. This is because besides LOS signal, Rx receives plenty of scattering path power caused by surrounding steal wall and chairs.

\section{B. RMS Delay Spread}

Delay Spread is an importance channel parameter in the system design since it determines cyclic prefix length of OFDM symbol. It also poses impact on frequency selectivity property of wireless channel.

RMS Delay Spread (DS) is defined as the square root of the second central moment and can be calculated as [8]:

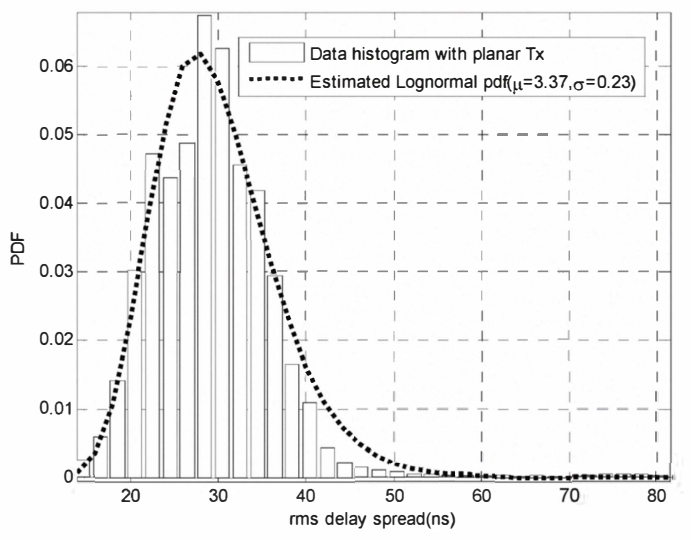

Fig. 7. Distribution of rms delay spread with planar antenna at $\mathrm{Tx}$.

$$
\sigma_{\tau}=\sqrt{\overline{\tau^{2}}-(\bar{\tau})^{2}}
$$

where

$$
\bar{\tau}=\frac{\sum_{\tau}\left|h_{\tau}\right|^{2} \tau}{\sum_{\tau}\left|h_{\tau}\right|^{2}}
$$

and

$$
\overline{\tau^{2}}=\frac{\sum_{\tau}\left|h_{\tau}\right|^{2} \tau^{2}}{\sum_{\tau}\left|h_{\tau}\right|^{2}}
$$

are the first and second moments of the instantaneous power-delay profile, respectively.

The measurement results with two different $\mathrm{Tx}$ configurations are shown in Fig. 6 and Fig.7. Here, we use lognormal model to fit the measurement data based on the LS criterion. The average values of DS with omni-directional and planar antennas are 30ns and 29ns, repectively, with almost the same variances. It can be concluded that the CDFs of DS are pretty similar in the closed environment yet with plenty of scatters, under these two Tx configurations.

\section{Ricean $\mathrm{K}$ - factor}

In the small closed environment, like the interior of high-speed train, there is a high probability when there is a dominant ray in the total complex path gain, with a zero-mean fluctuating component. In this case, the amplitude distributions of the taps can be modeled by Rice distribution [9]. The defining parameter of this distribution is the $\mathrm{K}$-factor, which is the power ratio of the fixed and fluctuating components.

To calculate $\mathrm{K}$-factor, the moment-method estimation is deployed [10-11], and K-factor is obtained as

$$
K=\frac{|V|^{2}}{\sigma^{2}} \text {, }
$$

where

$$
|V|^{2}=\sqrt{G_{m}^{2}-G_{v}^{2}}
$$

and

$$
\sigma^{2}=G_{m}-\sqrt{G_{m}^{2}-G_{v}^{2}} .
$$

The first moment $G_{m}$ is the average power gain and the second 


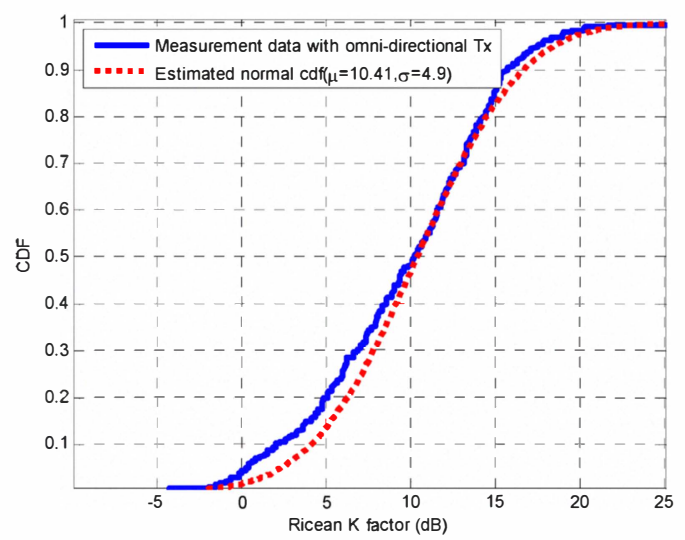

Fig. 8. Distribution of Ricean K-factor with omni-directional antenna at Tx.

moment $G_{v}$ is the RMS fluctuation of power gain.

In each measurement spot, we obtain one K-factor value. The blue line in Fig. 8 and Fig.9 shows the measurement results in decibel with omni-directional antenna and planar antenna, respectively. The distribution can be well modeled as normal distribution. The LS criterion is also applied.

The average values of $\mathrm{K}$-factor with omni-directional and planar antennas are $10.41 \mathrm{~dB}$ and $4.09 \mathrm{~dB}$, repectively, with similar variances. The reason for difference in average value is that, when omni-directional antenna as Tx is fixed on the ceil, it is more likely to receive LOS signal from $\mathrm{Rx}$ than when planar antenna is fixed on the wall. In fact, because of smaller closed envirionment, the average $\mathrm{K}$-factor value is also bigger than that in the LOS scenaro of InH in IMT-Adavanced channel model [12], where the Tx is also supposed to be omni-directional fixed on the ceil, although with similar variances.

\section{CONCLUSION}

Wideband channel measurements at $2.35 \mathrm{GHz}$ were carried out to facilitate future wideband high-speed train indoor network deployment. Two BS configures are considered and key channel parameters, such as Path Loss, Delay Spread and K-factor, are analyzed and modeled.

The empirical log-distance PL models are derived based on the measurement data. It is found that PL with planar antenna at $\mathrm{Tx}$ is $10 \mathrm{~dB}$ bigger than that with omni-directional antenna at $\mathrm{Tx}$. The latter is even less than that of free space. However, the results of RMS DS turn out to be quite similar in terms of mean value and variance under these two BS configurations. The result also shows that the distribution of RMS DS can be well fitted with lognormal distribution. The average value is about 30ns. Additionally, distributions of Ricean K-factor in decibel under these two BS configurations follow normal distribution, but the average values are pretty different, which are $10.41 \mathrm{~dB}$ and $4.09 \mathrm{~dB}$ with omni-directional antenna and plarnar antenna, respectively.

The channel properties analyzed and modeled in this paper is mainly for the indoor part of high-speed train communication. As to outdoor part, which is from BS to the antennas fixed on

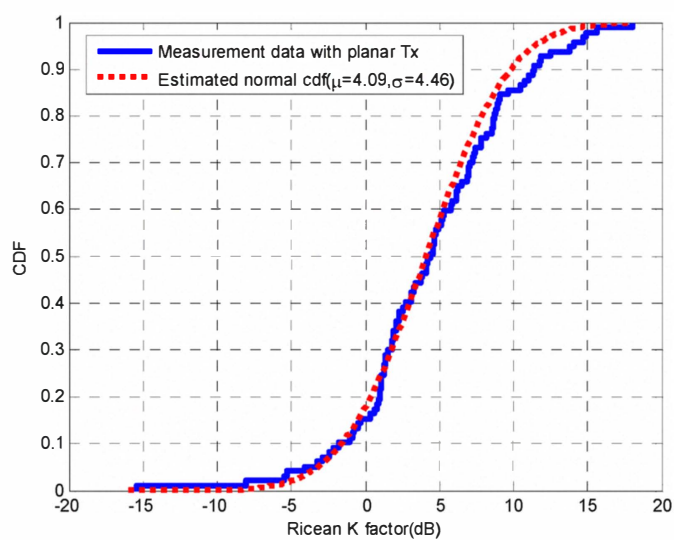

Fig. 9. Distribution of Ricean K-factor with planar antenna at Tx

the top of the train, future work will be carried out.

\section{ACKNOWLEDGEMENT}

The research is supported by China Important National Science and Technology Specific Projects with No. 2009ZX03007-003-01.

\section{REFERENCES}

[1] Webb, W. T., and Shenton, R. D., 'Pan-European Railway Communications: Where PMR and Cellular Meet', Electronics and Communications Engineering Journal, August 1994, pp. 195-202.

[2] Farrokh Abrishamkar and James Irvine, "Comparison of Current Solutions for the Provision of Voice Services to Passengers on High Speed Trains", Vehicular Technology Conference (VTC 2000), Tokyo, Japan, 15-18 May, 2000.

[3] 3GPP TS 36.104, "Base Station (BS) radio transmission and reception", V9.0.0

[4] 3GPP TS 36.101, "User Equipment (UE) radio transmission and reception", V9.3.0

[5] A. Stucki et. al., "PropSound System Specifications Document: Concept and Specifications," Elektrobit AG, Switzerland, Tech. Rep., 2001.

[6] V. Erceg, L. J. Greenstein, S. Y. Tjandra, S. R. Parkoff, A. Gupta, B. Kulic A. A. Julius, and R. Bianchi, "An empirically based path loss model for wireless channels in suburban environments," IEEE J. Sel. Areas Commun., vol. 17, pp. 1205-1211, Jul. 1999

[7] X. Gao, J. Zhang, G. Liu, D. Xu, P. Zhang, Y. Lu, and W. Dong, "Large-Scale Characteristics of $5.25 \mathrm{GHz}$ Based on Wideband MIMO Channel Measurements", IEEE Antennas and Wireless Propagation Letters, vol. 6, 2007

[8] T. S. Rappaport, Wireless Communications, Principles and Practice. Englewood Cliffs, NJ: Prentice-Hall, 1996

[9] S. O. Rice, "Statistical properties of a sine wave plus random noise," Bell Syst. Tech. J., vol. 27, pp. 109-157, Jan. 1948

[10] L. J. Greenstein, D. G. Michelson, and V. Erceg, "Moment-method estimation of the Ricean $K$-factor," IEEE Commun. Lett., vol. 3, no. 6, pp. 175-176, Jun. 1999.

[11] L. J. Greenstein, S. S. Ghassemzadeh, V. Erceg, D. G. Michelson, "Ricean K-Factors in Narrow-Band Fixed Wireless Channels: Theory, Experiments, and Statistical Models," IEEE Trans. on Vehicular Technology, vol. 58, NO. 8, Oct. 2009

[12] ITU-R Report M.2135, Guidelines for evaluation of radio interface technologies for IMT-Advanced, 2008-11, http://www.itu.int/publ/R-REP-M.2135-2008/en 